\title{
Respostas fisiológicas de frangos de corte industriais criados sob condições ambientais em Rio Branco - Acre
}

\author{
[Physiological responses of broiler chickens reared under the environmental conditions of Rio \\ Branco - Acre]
}

\section{"Artigo Científico/Scientific Article"}

\author{
Gilcineide Araújo Pires $^{1 *}$, Marcelo Bastos Cordeiro ${ }^{2}$, Antônia Mariana do Nascimento ${ }^{1}$, Suelen \\ Ferreira da Costa Rodrigues ${ }^{1}$, Francisco Cildomar da Silva Correia ${ }^{1}$, Henrique Jorge de Freitas ${ }^{1}$, \\ Edcarlos Miranda de Souza ${ }^{1}$
}

\author{
${ }^{1}$ Programa de Pós-Graduação em Sanidade e Produção Animal Sustentável na Amazônia Ocidental, Universidade \\ Federal do Acre, Rio Branco - AC, Brasil. \\ ${ }^{2}$ Instituto de Ciências Agrárias, Universidade Federal dos Vales do Jequitinhonha e Mucuri, Unaí-MG, Brasil. \\ *Autor para correspondência/Corresponding author: E-mail: gilce.pires@gmail.com
}

\begin{abstract}
Resumo
Esse estudo teve como objetivo avaliar as respostas fisiológicas de frangos de corte industriais e caracterizar as condições climáticas do ambiente interno de criação das aves em Rio Branco - Acre. Utilizou-se 280 aves de linhagens comercial (Cobb e Ross), distribuídas em delineamento inteiramente casualizado, em esquema de parcelas subdivididas, em que na parcela as linhagens foram consideradas como tratamentos primários e os períodos de coleta tratamento secundário, com 7 repetições e 10 aves por boxe. As variáveis ambientais analisadas foram: temperatura $\left({ }^{\circ} \mathrm{C}\right)$, umidade relativa (UR) e Índice de Temperatura de Globo Negro e Umidade (ITGU). Os parâmetros avaliados nas aves foram: Temperatura Média Corporal (TMC), Frequência Respiratória (FR) e Temperatura Cloacal (TCL). Os resultados demonstraram ocorrência de estresse por frio nas primeiras 3 semanas de vida das aves, diminuindo ao longo das semanas, sendo que a partir da quarta semana, o estresse por calor foi predominante, chegando a alcançar cerca de 70,4\% do tempo de exposição, considerando a temperatura $\left({ }^{\circ} \mathrm{C}\right)$ a UR e o ITGU, houve efeito do período para todas as variáveis estudadas. Não foi observada implicação das linhagens para nenhum dos parâmetros avaliados, exceto para a TMC entre machos das linhagens estudadas aos 8 dias de idade e de FR aos 43 dias de idade. As médias elevadas de TMC e FR indicaram desconforto das aves, já a TCL se manteve constante. Constatou-se que as variáveis climáticas se apresentaram fora da faixa de conforto térmico para frangos de corte, porém as aves conseguiram ajustar-se fisiologicamente, mantendo sua homeotermia.
\end{abstract}

Palavras-chave: conforto térmico; fisiologia; ITGU; microclima.

\begin{abstract}
The objective of this study was to evaluate the physiological responses of broiler chickens and to characterize the climatic conditions of the broiler breeding environment. A total of 280 commercial (Cobb and Ross) broilers were used, in a completely randomized design, in a subdivided plots scheme, in which the lineages were considered as primary treatments and the secondary treatment the collection periods, with 7 replicates and 10 replicates birds per box. The environmental variables analyzed were temperature $\left({ }^{\circ} \mathrm{C}\right)$, relative humidity (RU) and Black Globe Temperature and Humidity Index (GTHI). The parameters evaluated in the birds were: Body Average Temperature (ABT), Respiratory Rate (RR) and Cloacal Temperature (CLT). The results showed cold stress occurrence in the first 3 weeks of life of the birds, decreasing over the weeks, from the fourth week, heat stress was predominant, reaching about $70.4 \%$ of the exposure time, considering the temperature $\left({ }^{\circ} \mathrm{C}\right)$, a RU and the GTHI, it was observed an effect of the period for all variables studied. No implication of the lineages was observed for any of the evaluated parameters, except for ABT between males of the strains studied at 8 days of age and for RF at 43 days of age. The high averages of ABT and RF indicated discomfort of the birds, and the CLT remained constant. It was verified
\end{abstract}


that the climatic variables were outside the thermal comfort range for broilers, but the birds were able to adjust physiologically, maintaining their homeothermia.

Keywords: thermal comfort; physiology; GTHI; microclimate.

\section{Introdução}

O controle térmico em ambientes destinados à criação de aves é de fundamental importância para o sucesso da atividade de produção avícola em regiões onde predominam altas temperaturas, uma vez que frangos de corte apresentam sensibilidade às variações climáticas (Furlan, 2006; Nascimento et al., 2011).

As formas mais eficientes de trocas de calor pelas aves podem ocorrer de modo sensível (condução, radiação e convecção) nas primeiras semanas de vida, e de modo latente (respiração e evaporação cutânea) na fase adulta (Borges et al., 2003).

O mecanismo de troca de calor das aves só é eficiente quando a temperatura e a UR se encontram dentro de limites considerados como ideais para manutenção de seu conforto térmico (Cassuce, 2011), que nesse caso pode ser entendida como faixa de temperatura ambiente, em que as taxas metabólicas são mínimas e a homeotermia pode ser mantida com menor gasto de energia metabólica contida na ração fornecida (Nazareno et al., 2009; Nascimento et al., 2014).

Aspectos relacionados ao conforto térmico podem ter influência direta sobre: peso, idade, densidade de alojamento, alimentação, estado fisiológico, genética e manejo (Amaral et al., 2011). Quanto ao ambiente, Baêta e Souza (2010) salientam que elementos climáticos como temperatura, velocidade do vento, umidade relativa (UR) e radiação solar, podem ser mensurados pelo índice de Temperatura de Globo Negro e Umidade (ITGU), visto que estes fatores podem apresentar influências sobre as respostas produtivas e fisiológicas das aves.

A mensuração da Temperatura Cloacal (TCL) como um dos indicadores mais eficientes para avaliação da termorregulação corporal em aves de corte (Cotta, 2012; Bueno et al., 2014), haja vista que a temperatura superficial sofre maior influência do ambiente (Marchini et al., 2007; Nascimento et al., 2015) e a frequência respiratória (FR) pode ser influenciada pela perda de calor por evaporação (Amaral et al., 2011; Camargo et al., 2015).

O melhoramento genético de frangos de corte é uma realidade no setor avícola. No entanto, há necessidade de melhorar a adaptação desses animais às condições climáticas do Brasil para que se possa aperfeiçoar a produtividade (Moura et al., 2017).

Nesse contexto, o presente estudo teve como objetivo avaliar as respostas fisiológicas em frangos de corte, bem como caracterizar as condições climáticas do ambiente de criação desses indivíduos em Rio Branco - Acre.

\section{Material e Métodos}

\section{Local de estudo}

O estudo foi desenvolvido no galpão experimental do Setor de Avicultura da Universidade Federal do Acre - UFAC, Campus Rio Branco (Figura 1).

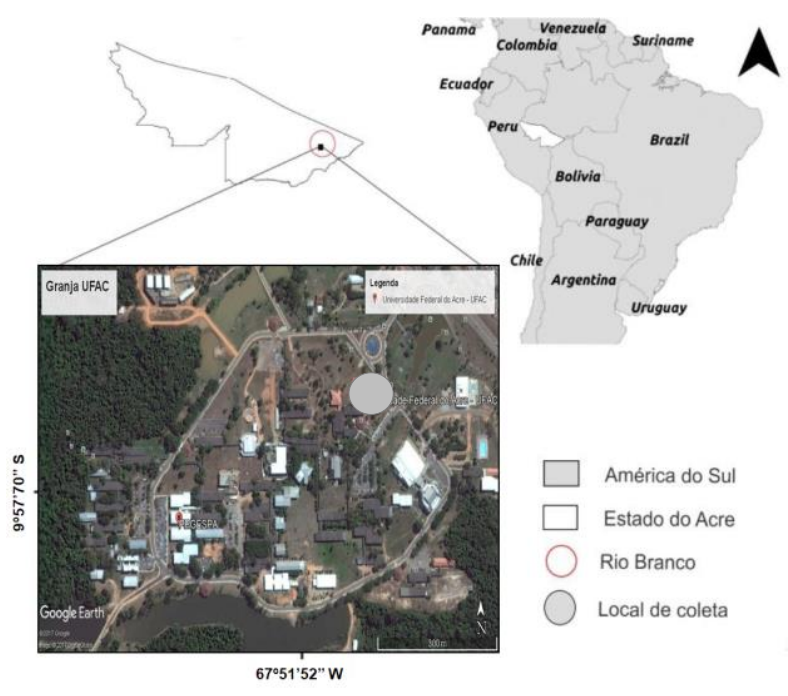

Figura 1. Local de realização do estudo e coleta de dados (Foto: Google Earth).

O galpão experimental possui $16 \mathrm{~m} \times 5 \mathrm{~m}$ (comprimento e largura, respectivamente), dividido em 32 boxes com dimensões de $2 \mathrm{~m} \times 1 \mathrm{~m}$ cada, estando disposto no sentido Leste-Oeste, com pé direito de $2,8 \mathrm{~m}$, coberto com telhas de alumínio e lanternim de fibrocimento. Possui piso e muretas laterais de concreto, pilares e tesoura de madeira, cercado com tela de arame, bem como as divisórias internas. 


\section{Clima}

De acordo com a classificação de Köppen (1931), o estado do Acre caracteriza-se por apresentar clima do tipo equatorial, quente e úmido, destacando-se duas estações distintas (chuvosa e seca). Um dos fatores característicos desta região é a elevada pluviosidade, compreendendo limites entre 1.600 a 2.750 $\mathrm{mm} / \mathrm{ano}$, com temperaturas médias variando entre $24,5^{\circ} \mathrm{C}$ e $32{ }^{\circ} \mathrm{C}$.

\section{Manejo zootécnico}

O manejo zootécnico adotado foi o proposto por Cobb-Vantress (2018) e Aviagen (2018), para criação de frangos de corte. A ração comercial fornecida foi à base de milho moído, farelo de soja e fubá de milho, formulada para atender às exigências nutricionais das aves em proteína, energia, cálcio e fósforo em cada fase de desenvolvimento das aves (inicial, crescimento e final) de acordo com Rostagno et al. (2005).

As aves foram criadas até 63 dias de idade no sistema convencional de criação, em piso coberto com maravalha como material de cama, em densidade de 5 aves $/ \mathrm{m}^{2}$, com água e ração à vontade.

\section{Obtenção das amostras}

As coletas dos dados foram realizadas em períodos distintos (manhã e tarde), utilizando-se 280 frangos de corte de linhagens comerciais (Cobb e Ross). O experimento ocorreu entre os meses de julho a setembro de 2016.

A avaliação dos parâmetros fisiológicos foi realizada analisando-se a temperatura retal, por meio de termômetro clínico digital, com precisão de $0,1{ }^{\circ} \mathrm{C}$, temperatura de superfície corporal através de sensor infravermelho digital com precisão de $\quad 0,5 \quad{ }^{\circ} \mathrm{C} \quad \mathrm{e} \quad(\mathrm{FR})$ avaliando $\mathrm{o}$ comportamento das aves (mov. $\mathrm{min}^{-1}$ ) a partir da segunda semana de vida, seguindo a metodologia proposta por Amaral et al. (2011). Os dados coletados foram armazenados em computador até o momento das análises.

\section{Análise dos dados}

Os dados fisiológicos coletados foram utilizados no cálculo da temperatura média da pele (TMP) e da temperatura média corporal (TMC) das aves, calculadas de acordo com a equação proposta por Richards (1971), considerando as temperaturas de superfície e a temperatura retal das aves:

$$
\begin{gathered}
T M P=(0,03 T C+0,70 T D+0,12 T A+0,06 \\
T C A+0,09 T P)
\end{gathered}
$$

Em ${ }^{\circ} \mathrm{C}$, em que: $\mathrm{TC}=$ temperatura de crista; $\mathrm{TD}=$ temperatura do dorso; $\mathrm{TA}=$ temperatura da asa; TCA = temperatura da cabeça e TP $=$ temperatura da pata.

$$
T M C=0,3 T M P+0,7 T R
$$

Em ${ }^{\circ} \mathrm{C}$, em que: $\mathrm{TMP}=$ Temperatura média da pele (TMP) e TR = temperatura retal.

As respostas fisiológicas foram tabuladas no programa da Microsoft Excel 2013. As análises estatísticas foram realizadas utilizando-se o programa Sisvar (Sistema para Análises Estatísticas), versão 5.6 (Ferreira, 2014) utilizouse $o$ teste $\mathrm{F}$ para indicar a diferença entre as médias.

As variáveis foram mensuradas utilizandose delineamento inteiramente ao acaso em parcelas subdivididas no tempo, em que a linhagem foi considerada o tratamento primário e o período o tratamento secundário, seguindo a metodologia descrita por Banzato e Kronka (2006).

As análises de variância seguiram os critérios de avaliação ao nível de $5 \%$ de significância. Neste caso, foi realizado o estudo dos efeitos dos fatores individualmente (linhagens e períodos), bem como o efeito da interação entre os mesmos.

O monitoramento da temperatura, umidade relativa do ar e temperatura de globo negro do galpão foi realizado por meio de Data Logger (modelo HOBO U12 - 012), com exatidão de \pm $0,35{ }^{\circ} \mathrm{C}$, em dois pontos medianos no interior do galpão com intervalo de 15 minutos durante todo o período experimental.

Com os valores, foram calculados o ITGU, determinado pela equação proposta por Buffington et al. (1981), que é dada por:

$$
I T G U=T g n+0,36(T p o)-330,08
$$

Em que Tgn é a Temperatura de globo negro e Tpo é a Temperatura de ponto de orvalho.

\section{Resultados}

Foi observado que a variação de temperatura ao longo da criação das aves encontrou-se fora da faixa de conforto térmico dos 
animais, entre 22 a $27^{\circ} \mathrm{C}$. $\mathrm{O}$ valor de temperatura encontrado no galpão, durante o período de criação das aves, apresentou intensa variação, expondo os animais ao estresse térmico por frio nas duas primeiras semanas e estresse térmico por calor, a partir da terceira semana (Figura 2).

Para o ITGU, a maior parte dos valores obtidos indicaram ambiente fora da zona de conforto para os frangos avaliados. Neste estudo foi verificado que o período mais crítico ocorreu a partir da quarta semana de vida das aves (Figura $3)$.

Também foi observada a ocorrência em porcentagem do período de tempo que as aves passaram em estresse calórico e conforto térmico. $\mathrm{O}$ estresse por frio se deu nas primeiras 3 semanas de vida, diminuindo gradativamente no decorrer do estudo, sendo que a partir da quarta semana, o estresse por calor foi predominante, chegando a alcançar cerca de $70,4 \%$ do tempo de exposição, com relação à temperatura do ar $\left({ }^{\circ} \mathrm{C}\right)$ e mais de 95,6\% para ITGU (Tabela 1 ).

Com relação à UR, foi observada uma amplitude considerável, indo de valores de 35\% até cerca de $90 \%$ para todo o período experimental (Figura 4).

$\mathrm{Na}$ Tabela 2 estão apresentadas as condições de ambiente seco, úmido e conforto, de acordo com o tempo transcorrido em cada semana de vida dos animais.

Observa-se que a condição seca é considerável na primeira e segunda semana de vida dos pintinhos. Este fator afeta principalmente o sistema respiratório das aves.

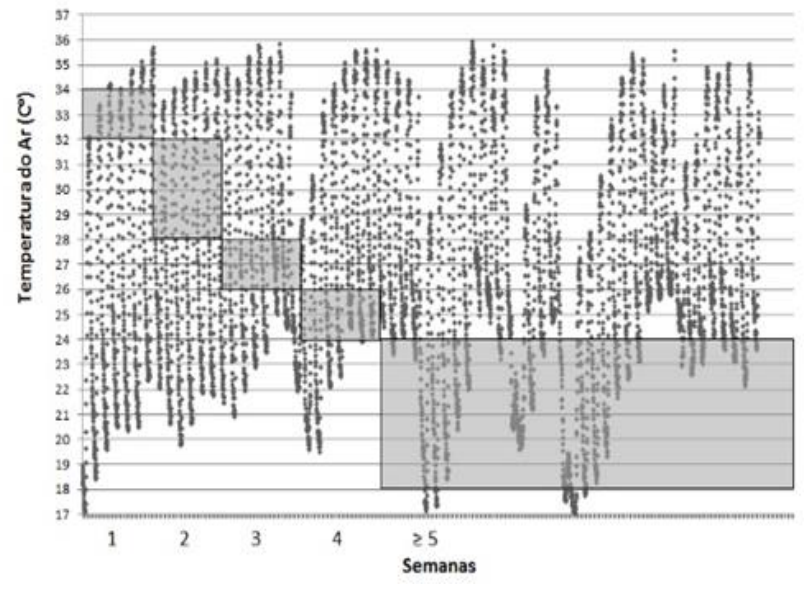

Figura 2. Temperatura $\left({ }^{\circ} \mathrm{C}\right)$ no interior do galpão, durante o período estudado.

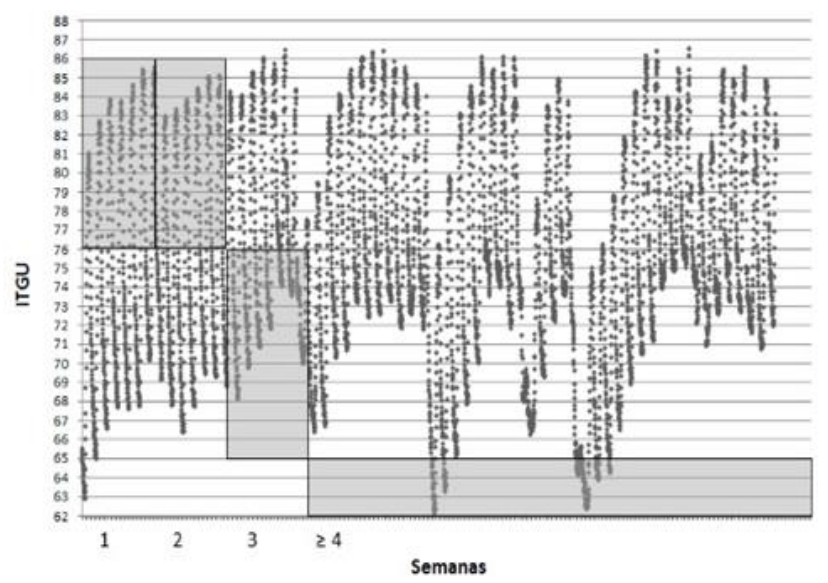

Figura 3. Índice de temperatura do globo negro e umidade (ITGU) no interior do galpão, durante as semanas de vida das aves.

Tabela 1. Ocorrência de condições de estresse calórico e conforto térmico, de acordo com o Índice de Temperatura do Globo Negro e Umidade (ITGU) e a temperatura.

\begin{tabular}{|c|c|c|c|c|c|c|c|c|c|c|}
\hline \multirow{3}{*}{ Condição } & \multicolumn{10}{|c|}{ Porcentagem de ocorrência (\%) } \\
\hline & \multicolumn{2}{|c|}{ Semana 1} & \multicolumn{2}{|c|}{ Semana 2} & \multicolumn{2}{|c|}{ Semana 3} & \multicolumn{2}{|c|}{ Semana 4} & \multicolumn{2}{|c|}{$\geq$ Semana 5} \\
\hline & ITGU & Temp. & ITGU & Temp. & ITGU & Temp. & ITGU & Temp. & ITGU & Temp. \\
\hline Calor & 0,0 & 8,6 & 0,0 & 23,8 & 46,5 & 39,6 & 95,6 & 58,9 & 95,6 & 70,4 \\
\hline Frio & 56,8 & 79,9 & 58,7 & 59,6 & 0,0 & 45,9 & 4,5 & 21,6 & 4,5 & 3,1 \\
\hline Conforto térmico & 43,2 & 11,5 & 41,3 & 16,5 & 53,5 & 14,5 & 0,0 & 19,5 & 0,0 & 26,5 \\
\hline
\end{tabular}

*Os valores das ocorrências são comparados de acordo com os dados da coluna.

Tabela 2. Ocorrência de condição seca, úmida e conforto, de acordo com a umidade relativa do ar (UR).

\begin{tabular}{cccccc}
\hline \multirow{2}{*}{ Condição } & \multicolumn{5}{c}{ Porcentagem de ocorrência (\%) } \\
\cline { 2 - 6 } & Semana 1 & Semana 2 & Semana 3 & Semana 4 & 2 Semana 5 \\
\hline Úmida & 37,4 & 20,9 & 40,1 & 37,3 & 45,8 \\
Seca & 33,7 & 40,2 & 25,8 & 32,4 & 24,8 \\
Conforto & 28,9 & 38,9 & 34,1 & 30,3 & 29,4 \\
\hline
\end{tabular}

*Os valores das ocorrências são comparados de acordo com os dados da coluna. 
A partir da quarta e quinta semana de vida, a condição úmida do ambiente é superior, sendo este fator crucial para a baixa eficiência da perda de calor latente pela ave. Com o aumento da UR, o mecanismo de ofegação do frango perde sua eficiência na perda de calor. Uma vez que se baseia em troca de calor latente, quanto mais úmido estiver o ambiente, menos água o animal consegue evaporar.

Realizou-se análise de variância para os dois fatores: linhagens e períodos. A fonte de variação linhagem não apresentou efeito significativo para nenhuma das variáveis avaliadas (Temperatura Média Corporal, Frequência Respiratória e Temperatura Cloacal), a interação não apresentou resultado significativo para nenhuma das variáveis analisadas e o período foi o único que apresentou resultados significativos, de modo que as médias apresentadas nas tabelas $(4,6$ e 8) são referentes à média comum para as linhagens Cobb e Ross.

A TMC das aves das linhagens Cobb e Ross relacionando os sexos em comparação aos dias de vida durante o período de 08 a 61 dias, respectivamente, está expressa na Tabela 3.

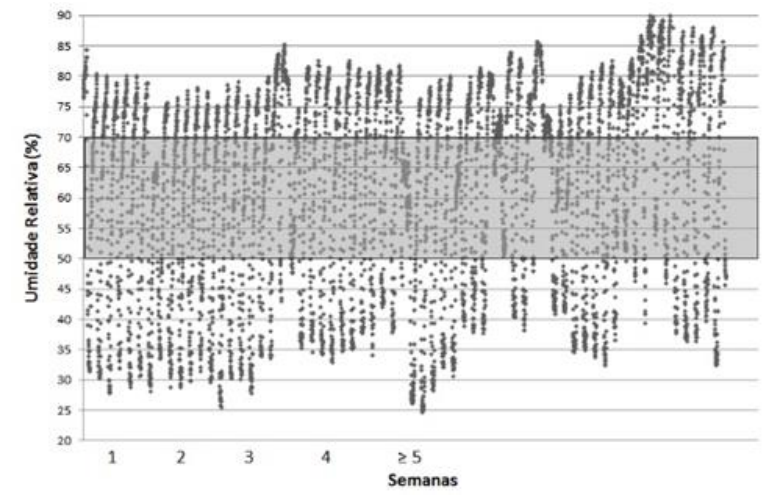

Figura 4. Umidade relativa (UR) do ar no interior do galpão no decorrer do estudo.

Tabela 3. Temperatura Média Corporal (TMC) em ${ }^{\circ} \mathrm{C}$ das aves relacionando o sexo em função das linhagens*

\begin{tabular}{ccccccc}
\hline \multirow{2}{*}{ Idade (dias) } & \multicolumn{3}{c}{ Fêmeas } & \multicolumn{3}{c}{ Machos } \\
\cline { 2 - 7 } & Cobb & Ross & $\begin{array}{c}\text { Erro } \\
\text { Padrão }( \pm)\end{array}$ & Cobb & Ross & $\begin{array}{c}\text { Erro } \\
\text { Padrão }( \pm)\end{array}$ \\
\hline 08 & $38,33 \mathrm{a}$ & $38,53 \mathrm{a}$ & 0,099 & $38,60 \mathrm{~b}$ & $38,81 \mathrm{a}$ & 0,067 \\
15 & $38,89 \mathrm{a}$ & $39,01 \mathrm{a}$ & 0,097 & $39,01 \mathrm{a}$ & $38,91 \mathrm{a}$ & 0,116 \\
22 & $38,76 \mathrm{a}$ & $38,64 \mathrm{a}$ & 0,133 & $38,70 \mathrm{a}$ & $38,83 \mathrm{a}$ & 0,181 \\
29 & $38,93 \mathrm{a}$ & $38,66 \mathrm{a}$ & 0,121 & $39,13 \mathrm{a}$ & $39,22 \mathrm{a}$ & 0,130 \\
36 & $39,01 \mathrm{a}$ & $39,08 \mathrm{a}$ & 0,134 & $39,38 \mathrm{a}$ & $39,15 \mathrm{a}$ & 0,094 \\
43 & $39,12 \mathrm{a}$ & $38,75 \mathrm{a}$ & 0,138 & $38,94 \mathrm{a}$ & $39,11 \mathrm{a}$ & 0,157 \\
50 & $39,01 \mathrm{a}$ & $39,08 \mathrm{a}$ & 0,134 & $39,37 \mathrm{a}$ & $39,15 \mathrm{a}$ & 0,094 \\
57 & $39,27 \mathrm{a}$ & $39,19 \mathrm{a}$ & 0,126 & $39,42 \mathrm{a}$ & $39,21 \mathrm{a}$ & 0,206 \\
61 & $39,27 \mathrm{a}$ & $39,20 \mathrm{a}$ & 0,126 & $39,42 \mathrm{a}$ & $39,22 \mathrm{a}$ & 0,206 \\
\hline
\end{tabular}

*Médias seguidas de letras diferentes na linha diferem significativamente entre si, pelo teste $\mathrm{F}$ ao nível de 5\% de probabilidade.

Analisando a influência das linhagens nos valores de TMC, observa-se diferença significativa de TMC apenas na primeira semana de vida das aves, aos 8 dias de idade $(\mathrm{P}<0,05)$, onde machos de linhagem Ross apresentaram maior média de temperatura corporal, comparado a linhagem Cobb.

Observa-se que em relação ao sexo, para fêmeas, houve variação de menor TMC aos 8 dias $\operatorname{com} 38,33{ }^{\circ} \mathrm{C}( \pm 0,099 \mathrm{EP})$ e maior com $39,27{ }^{\circ} \mathrm{C}$ $( \pm 0,126 \mathrm{EP})$ aos 57 e 61 dias. Para os machos, menor TMC aos 8 dias, com $38,60{ }^{\circ} \mathrm{C}( \pm 0,067 \mathrm{EP})$ e maior com $39,42{ }^{\circ} \mathrm{C}( \pm 0,206 \mathrm{EP})$ aos 57 e 61 dias.

Quanto à interação entre TMC x período do dia, observa-se na Tabela 4 que houve diferenças significativas $(\mathrm{P}<0,05)$ tanto para fêmeas, quanto para machos, sendo que as temperaturas corporais do período da manhã apresentaram-se menores.
Fato este, justifica-se pelo período da manhã apresentar temperatura ambiente mais amena.

À tarde o gradiente de temperatura entre o ambiente e a ave diminui, fazendo com que o frango não consiga dissipar calor com a mesma eficiência. Isto faz com que a ave retenha calor, podendo aumentar a sua temperatura basal.

Quando este gradiente de temperatura diminui, o mecanismo latente de troca de calor (ofegação) se torna o mais eficiente, pois se utiliza da troca de calor evaporativa. No entanto, esse mecanismo aumenta a taxa respiratória da ave, podendo ocorrer transtornos fisiológicos indesejáveis.

As médias das variáveis fisiológicas para FR diferiu $(\mathrm{P}<0,05)$ entre as linhagens (Tabela 5) aos 43 dias para os machos de linhagem Cobb, apresentando média de FR 102,28 mov/min $( \pm 3,099 \mathrm{EP})$. 
Tabela 4. Temperatura Média Corporal (TMC) em ${ }^{\circ} \mathrm{C}$ das aves relacionando-as ao período do dia (médias comuns para as duas linhagens) *

\begin{tabular}{ccccccc}
\hline \multirow{2}{*}{ Idade (dias) } & \multicolumn{3}{c}{ Fêmeas } & \multicolumn{3}{c}{ Machos } \\
\cline { 2 - 7 } & Manhã & Tarde & $\begin{array}{c}\text { Erro } \\
\text { Padrão }( \pm)\end{array}$ & Manhã & Tarde & $\begin{array}{c}\text { Erro } \\
\text { Padrão }( \pm)\end{array}$ \\
\hline 08 & $36,67 \mathrm{~b}$ & $40,20 \mathrm{a}$ & 0,111 & $37,06 \mathrm{~b}$ & $40,35 \mathrm{a}$ & 0,093 \\
15 & $37,53 \mathrm{~b}$ & $40,36 \mathrm{a}$ & 0,141 & $37,62 \mathrm{~b}$ & $40,30 \mathrm{a}$ & 0,090 \\
22 & $38,64 \mathrm{a}$ & $38,75 \mathrm{a}$ & 0,158 & $37,63 \mathrm{~b}$ & $39,90 \mathrm{a}$ & 0,116 \\
29 & $37,51 \mathrm{~b}$ & $40,08 \mathrm{a}$ & 0,193 & $38,06 \mathrm{~b}$ & $40,29 \mathrm{a}$ & 0,166 \\
36 & $37,61 \mathrm{~b}$ & $40,47 \mathrm{a}$ & 0,118 & $37,88 \mathrm{~b}$ & $40,64 \mathrm{a}$ & 0,112 \\
43 & $37,90 \mathrm{~b}$ & $39,96 \mathrm{a}$ & 0,169 & $37,96 \mathrm{~b}$ & $40,08 \mathrm{a}$ & 0,155 \\
50 & $37,61 \mathrm{~b}$ & $40,47 \mathrm{a}$ & 0,118 & $37,88 \mathrm{~b}$ & $40,64 \mathrm{a}$ & 0,112 \\
57 & $38,49 \mathrm{~b}$ & $39,97 \mathrm{a}$ & 0,170 & $38,44 \mathrm{~b}$ & $40,20 \mathrm{a}$ & 0,076 \\
61 & $38,49 \mathrm{~b}$ & $39,97 \mathrm{a}$ & 0,170 & $38,44 \mathrm{~b}$ & $40,20 \mathrm{a}$ & 0,076 \\
\hline
\end{tabular}

*Médias seguidas de letras diferentes na linha, diferem significativamente entre si, pelo teste $\mathrm{F}$ ao nível de $5 \%$ de probabilidade.

Tabela 5. Média da frequência respiratória (mov/min) das aves comparada com a linhagem e sexo das aves em função da idade (dia)*

\begin{tabular}{ccccccc}
\hline \multirow{2}{*}{ Idade (dias) } & \multicolumn{3}{c}{ Fêmeas } & \multicolumn{2}{c}{ Machos } \\
\cline { 2 - 7 } & Cobb & Ross & $\begin{array}{c}\text { Erro } \\
\text { Padrão }( \pm)\end{array}$ & Cobb & Ross & $\begin{array}{c}\text { Erro } \\
\text { Padrão }( \pm)\end{array}$ \\
\hline 08 & $50,57 \mathrm{a}$ & $52,57 \mathrm{a}$ & 2,885 & $52,57 \mathrm{a}$ & $54,57 \mathrm{a}$ & 3,061 \\
15 & $79,71 \mathrm{a}$ & $68,85 \mathrm{a}$ & 6,735 & $80,85 \mathrm{a}$ & $84,28 \mathrm{a}$ & 7,617 \\
22 & $58,00 \mathrm{a}$ & $69,14 \mathrm{a}$ & 5,829 & $89,42 \mathrm{a}$ & $70,85 \mathrm{a}$ & 7,693 \\
29 & $68,57 \mathrm{a}$ & $76,85 \mathrm{a}$ & 5,269 & $68,85 \mathrm{a}$ & $80,57 \mathrm{a}$ & 4,816 \\
36 & $40,81 \mathrm{a}$ & $39,25 \mathrm{a}$ & 0,938 & $103,14 \mathrm{a}$ & $97,42 \mathrm{a}$ & 4,845 \\
43 & $102,85 \mathrm{a}$ & $108,28 \mathrm{a}$ & 6,262 & $102,28 \mathrm{a}$ & $91,71 \mathrm{~b}$ & 3,099 \\
50 & $94,85 \mathrm{a}$ & $100,28 \mathrm{a}$ & 7,226 & $99,71 \mathrm{a}$ & $92,28 \mathrm{a}$ & 4,688 \\
57 & $39,27 \mathrm{a}$ & $39,19 \mathrm{a}$ & 8,771 & $99,42 \mathrm{a}$ & $93,14 \mathrm{a}$ & 4,634 \\
61 & $39,27 \mathrm{a}$ & $39,20 \mathrm{a}$ & 8,771 & $99,41 \mathrm{a}$ & $92,42 \mathrm{a}$ & 4,634 \\
\hline
\end{tabular}

*Médias seguidas de letras diferentes na linha diferem significativamente entre si, pelo teste $\mathrm{F}$ ao nível de $5 \%$ de probabilidade.

Nesse estudo as aves encontravam-se fora da faixa de conforto térmico, pois somente as fêmeas apresentaram diminuição perceptível nos valores de FR nas duas últimas análises e na primeira semana de vida, a FR apresentou menor valor de 50,57 $\mathrm{mov} / \mathrm{min}( \pm 2,885 \mathrm{EP})$.

Os resultados mostram que as médias de FR variam de $39,19 \mathrm{mov} / \mathrm{min}( \pm 8,771 \mathrm{EP})$ a 108,28 $\mathrm{mov} / \mathrm{min}( \pm 6,262 \mathrm{EP})$ entre as fêmeas e 52,27 $\mathrm{mov} / \mathrm{min} \quad( \pm 2,885 \mathrm{EP}) \quad$ a $\quad 103,14 \quad \mathrm{mov} / \mathrm{min}$ $( \pm 4,845 \mathrm{EP})$ entre os machos.

Quanto à interação de FR das linhagens $\mathrm{x}$ período do dia, a FR diferiu $(\mathrm{P}<0,05)$ entre os períodos da manhã e da tarde. Com exceção da primeira semana, aos 8 dias de idade, as aves apresentaram semelhança nos valores de FR, indicando que até esta fase, nos dois períodos, as aves apresentavam o mesmo comportamento com relação as condições ambientais (Tabela 6).

Tabela 6. Frequência Respiratória (FR) em mov/min das aves relacionando-as ao período do dia (médias comuns para as duas linhagens)*

\begin{tabular}{ccccccc}
\hline \multirow{2}{*}{ Idade (dias) } & \multicolumn{3}{c}{ Fêmeas } & \multicolumn{2}{c}{ Machos } \\
\cline { 2 - 7 } & Manhã & Tarde & $\begin{array}{c}\text { Erro } \\
\text { Padrão }( \pm)\end{array}$ & Manhã & Tarde & $\begin{array}{c}\text { Erro } \\
\text { Padrão }( \pm)\end{array}$ \\
\hline 08 & $46,57 \mathrm{a}$ & $56,57 \mathrm{a}$ & 3,265 & $53,42 \mathrm{a}$ & $53,71 \mathrm{a}$ & 2,432 \\
15 & $47,71 \mathrm{~b}$ & $100,85 \mathrm{a}$ & 7,477 & $55,14 \mathrm{~b}$ & $110,00 \mathrm{a}$ & 8,055 \\
22 & $47,71 \mathrm{~b}$ & $79,42 \mathrm{a}$ & 6,130 & $46,00 \mathrm{~b}$ & $114,28 \mathrm{a}$ & 7,901 \\
29 & $44,85 \mathrm{~b}$ & $100,57 \mathrm{a}$ & 4,344 & $45,42 \mathrm{~b}$ & $104,00 \mathrm{a}$ & 5,287 \\
36 & $38,28 \mathrm{~b}$ & $41,77 \mathrm{a}$ & 1,058 & $40,57 \mathrm{~b}$ & $160,00 \mathrm{a}$ & 6,475 \\
43 & $57,14 \mathrm{~b}$ & $153,71 \mathrm{a}$ & 3,712 & $52,00 \mathrm{~b}$ & $142,00 \mathrm{a}$ & 6,331 \\
50 & $39,71 \mathrm{~b}$ & $155,42 \mathrm{a}$ & 7,477 & $47,42 \mathrm{~b}$ & $145,14 \mathrm{a}$ & 5,508 \\
61 & $38,43 \mathrm{~b}$ & $39,97 \mathrm{a}$ & 6,396 & $47,42 \mathrm{~b}$ & $145,14 \mathrm{a}$ & 6,075 \\
\hline
\end{tabular}

*Médias seguidas de letras diferentes na linha diferem significativamente entre si, pelo teste $\mathrm{F}$ ao nível de $5 \%$ de probabilidade. 
Os menores valores de FR das aves foram observados no período da manhã para os dois sexos. O valor mínimo de FR no período da manhã foi de $38,28 \mathrm{mov} / \mathrm{min}( \pm 1,058 \mathrm{EP})$ e a máxima de $57,14 \mathrm{mov} / \mathrm{min}( \pm 3,712 \mathrm{EP})$. No período da tarde, apresentaram valor mínimo de FR de 39,97 mov/min $( \pm 6,396 \mathrm{EP})$ e máxima de
$160 \mathrm{mov} / \mathrm{min}( \pm 6,475 \mathrm{EP})$. Devem-se às condições térmicas gerais observadas nos dois períodos (manhã e tarde), conforto térmico pela manhã e estresse por calor à tarde.

$\mathrm{Na}$ Tabela 7, encontram-se os dados de TCL das aves em função do sexo e linhagem de acordo com a idade.

Tabela 7. Temperatura Cloacal (TCL) em ${ }^{\circ} \mathrm{C}$ das aves em função do sexo e linhagem de acordo com a idade*

\begin{tabular}{ccccccc}
\hline \multirow{2}{*}{ Idade (dias) } & \multicolumn{3}{c}{ Fêmeas } & \multicolumn{3}{c}{ Machos } \\
\cline { 2 - 7 } & Cobb & Ross & $\begin{array}{c}\text { Erro } \\
\text { Padrão }( \pm)\end{array}$ & Cobb & Ross & $\begin{array}{c}\text { Erro } \\
\text { Padrão }( \pm)\end{array}$ \\
\hline 08 & $39,90 \mathrm{a}$ & $40,09 \mathrm{a}$ & 0,120 & $40,07 \mathrm{a}$ & $40,22 \mathrm{a}$ & 0,072 \\
15 & $40,32 \mathrm{a}$ & $40,46 \mathrm{a}$ & 0,100 & $40,53 \mathrm{a}$ & $40,43 \mathrm{a}$ & 0,130 \\
22 & $40,55 \mathrm{a}$ & $40,45 \mathrm{a}$ & 0,151 & $40,32 \mathrm{a}$ & $40,54 \mathrm{a}$ & 0,146 \\
29 & $40,79 \mathrm{a}$ & $40,66 \mathrm{a}$ & 0,121 & $40,70 \mathrm{a}$ & $40,81 \mathrm{a}$ & 0,138 \\
36 & $41,17 \mathrm{a}$ & $41,15 \mathrm{a}$ & 0,127 & $41,12 \mathrm{a}$ & $41,15 \mathrm{a}$ & 0,103 \\
43 & $41,21 \mathrm{a}$ & $41,13 \mathrm{a}$ & 0,169 & $40,99 \mathrm{a}$ & $41,04 \mathrm{a}$ & 0,161 \\
50 & $41,17 \mathrm{a}$ & $41,15 \mathrm{a}$ & 0,127 & $41,12 \mathrm{a}$ & $41,15 \mathrm{a}$ & 0,103 \\
57 & $41,25 \mathrm{a}$ & $41,42 \mathrm{a}$ & 0,162 & $41,76 \mathrm{a}$ & $41,38 \mathrm{a}$ & 0,203 \\
61 & $41,27 \mathrm{a}$ & $41,20 \mathrm{a}$ & 0,162 & $41,76 \mathrm{a}$ & $41,38 \mathrm{a}$ & 0,203 \\
\hline
\end{tabular}

*Médias seguidas com letras iguais na linha, não diferem significativamente entre si, pelo teste $\mathrm{F}$ ao nível de $5 \%$ de probabilidade.

De acordo com o teste $\mathrm{F}$, a TCL não diferiu estatisticamente $(\mathrm{P}>0,05)$ para ambas as linhagens nos diferentes sexos, apresentando pouca variação entre as mesmas. As médias de TCL variaram em torno de $40,8^{\circ} \mathrm{C}( \pm 0,138 \mathrm{EP})$.

As médias de TCL dentro da faixa admissível para conforto térmico indicam que os mecanismos de dissipação de calor conseguiram manter o animal na faixa de homeotermia aceitável.

Com relação às médias de TCL das aves, observou-se que no período da tarde foram superiores ao da manhã $(\mathrm{P}<0,05)$, para machos e fêmeas, a partir do 22 dia (Tabela 8).

Neste estudo, machos apresentaram TCL superiores às encontradas nas fêmeas.

Tabela 8. Temperatura Cloacal (TCL) em ${ }^{\circ} \mathrm{C}$ das aves relacionando-as ao período do dia (médias comuns para as duas linhagens)*

\begin{tabular}{ccccccc}
\hline \multirow{2}{*}{ Idade (dias) } & \multicolumn{3}{c}{ Fêmeas } & \multicolumn{3}{c}{ Machos } \\
\cline { 2 - 7 } & Manhã & Tarde & $\begin{array}{c}\text { Erro } \\
\text { Padrão }( \pm)\end{array}$ & Manhã & Tarde & $\begin{array}{c}\text { Erro } \\
\text { Padrão }( \pm)\end{array}$ \\
\hline 08 & $39,57 \mathrm{~b}$ & $40,41 \mathrm{a}$ & 0,141 & $39,82 \mathrm{~b}$ & $40,48 \mathrm{a}$ & 0,110 \\
15 & $39,70 \mathrm{~b}$ & $41,09 \mathrm{a}$ & 0,143 & $39,85 \mathrm{~b}$ & $41,11 \mathrm{a}$ & 0,102 \\
22 & $40,06 \mathrm{~b}$ & $40,92 \mathrm{a}$ & 0,123 & $39,75 \mathrm{~b}$ & $41,12 \mathrm{a}$ & 0,159 \\
29 & $39,91 \mathrm{~b}$ & $41,54 \mathrm{a}$ & 0,144 & $40,21 \mathrm{~b}$ & $41,30 \mathrm{a}$ & 0,140 \\
36 & $40,55 \mathrm{~b}$ & $41,77 \mathrm{a}$ & 0,096 & $40,40 \mathrm{~b}$ & $41,87 \mathrm{a}$ & 0,161 \\
43 & $40,74 \mathrm{~b}$ & $41,60 \mathrm{a}$ & 0,139 & $40,33 \mathrm{~b}$ & $41,70 \mathrm{a}$ & 0,125 \\
50 & $40,55 \mathrm{~b}$ & $41,77 \mathrm{a}$ & 0,096 & $40,40 \mathrm{~b}$ & $41,87 \mathrm{a}$ & 0,161 \\
61 & $40,77 \mathrm{~b}$ & $41,90 \mathrm{a}$ & 0,105 & $40,90 \mathrm{~b}$ & $42,24 \mathrm{a}$ & 0,193 \\
\hline
\end{tabular}

*Médias seguidas de letras diferentes na linha diferem significativamente entre si, pelo teste $\mathrm{F}$ ao nível de $5 \%$ de probabilidade.

\section{Discussão}

O monitoramento da temperatura durante o período de criação dos frangos de corte foi considerada significativa para caracterização de um ambiente confortável, corroborando com os resultados encontrados por Cassuce (2011), que verificou situações de conforto térmico no ambiente interno do galpão para pintinhos na primeira semana de vida com temperaturas variando entre 32 e $34{ }^{\circ} \mathrm{C}$.

O ITGU fora da zona de conforto para as aves caracteriza-se com valores de ITGU superiores a 77 como apresentaram os dados coletados por Amaral et al. (2011), visto que em outro estudo realizado por Gomes et al. (2011), os valores de ITGU considerados limites de conforto térmico são 69 para inferior e 77 para superior. 
Com relação à umidade relativa do ar (UR), notou-se uma variação relevante ao longo da criação das aves. De acordo com Baêta e Souza (2010), a temperatura, umidade, velocidade do vento e radiação solar, são os fatores climáticos que mais afetam os animais, por apresentarem forte influência em seu desempenho zootécnico, constituindo uma das principais causas de perdas produtivas em climas tropicais. Por esse motivo, nas três primeiras semanas de vida das aves o galpão fica o tempo inteiro envolvido por cortinas para que não haja interferência nos índices de UR no interior do galpão, uma vez que Tinôco e Gates (2005), consideram a faixa de 50 a $70 \%$ de UR ideal para frangos de corte.

Nesse sentido, a UR do ar destaca-se como o segundo fator determinante na produção animal (Abreu et al., 2012). Quando alcança valores abaixo de $50 \%$, pode ocasionar doenças respiratórias e membranas ressecadas, quando ultrapassam 70\%, os animais apresentam dificuldades para dissipar calor latente através da evaporação e da ofegação, pois com o ar saturado, a pressão de vapor d'água diminui (Pires e Campos, 2004; Baêta e Souza, 2010). Assim, a capacidade das aves em tolerar o calor está diretamente relacionada com o nível de UR.

$\mathrm{Na}$ avaliação fisiológica das aves, inicialmente verificando a Temperatura Média Corporal (TMC) das linhagens Cobb e Ross relacionando os sexos em comparação aos dias de vida, observou-se variação de TMC. De acordo com Bridi (2010), as aves, apresentam temperatura corporal de $41,1{ }^{\circ} \mathrm{C}$ em zona de conforto térmico.

Abreu et al. (2012) verificaram oscilação nos valores de temperatura superficial, com comportamento semelhante ao da temperatura ambiente. Observaram ainda, assim como Cangar et al. (2008) e Bueno et al. (2014), que aos 35 dias de idade das aves as temperaturas médias corporais elevaram-se a um nível máximo e decaíram até os 42 dias de idade, concluindo que a TMC diminui à medida que as aves ficam mais velhas, pois nessa fase seu sistema termorregulador já está desenvolvido, o que demanda uma condição de conforto térmico.

Quanto à interação entre TMC x período do dia, Lin et al. (2005) indicaram que a pouca diferença entre a temperatura ambiente e a temperatura da superfície corporal pode ser parcialmente responsável pelo aumento da temperatura corporal, principalmente em ambiente de temperatura elevada.

Giloh et al. (2012) verificaram um elevado coeficiente de correlação entre as temperaturas da superfície do pintinho e as variáveis ambientais, confirmando que a temperatura, a UR e a circulação do vento são os principais fatores que afetam o desempenho das aves. Pela manhã, os mecanismos sensíveis de troca de calor como condução, convecção e radiação apresentam maior eficiência no processo de dissipação de calor das aves. A troca de calor sensível é mais eficiente quando existe um maior gradiente de temperatura entre o animal e o ambiente (Souza et al., 2005).

As variáveis fisiológicas para FR entre as linhagens apresentaram diferenças somente aos 43 dias, evidenciado aos machos, que possuem a taxa de metabolismo mais ativa do que as fêmeas. Segundo Marchini et al. (2007), a FR reduz com a idade para as aves em termoneutralidade. $\mathrm{Na}$ primeira semana de vida, aves em situação de conforto, apresentam FR em torno de 48 $\mathrm{mov} / \mathrm{min}$.

De acordo com Nazareno et al. (2009), quando a FR apresenta valores elevados, entendese que a temperatura do ar ultrapassa o limite crítico superior para as aves, acarretando em aumento da temperatura corporal, devido ao calor armazenado.

Quanto à interação de FR das linhagens x período do dia, Costa et al. (2012) avaliaram os efeitos do ambiente sobre os indicadores fisiológicos de frango de corte, de linhagem Cobb, nos diferentes horários: 7, 10, 13,16 e 19 horas, e concluíram que as variáveis climáticas se mantiveram fora da faixa de considerada confortável para as aves, vindo a desencadear dissipação de calor por evaporação através do trato respiratório, sendo às 13 horas o horário que representou a maior média de (FR) com 58,96 mov/min e TCL em torno de $41^{\circ} \mathrm{C}$.

A mensuração da TCL indica com mais precisão a temperatura interna do indivíduo, por ser um mecanismo utilizado para liberação de calor armazenado (Costa et al., 2012). Marchini et al. (2007) consideram para TCL o valor de 41,4 ${ }^{\circ} \mathrm{C}$ para condições de termoneutralidade, assemelhando-se aos resultados deste estudo.

Como verificado neste estudo, os machos apresentaram TCL acima das observadas em fêmeas. Bueno et al. (2014) sugerem que isso deve ocorrer devido a maior atividade metabólica 
dos machos, que resulta em maior produção de calor.

\section{Conclusão}

As condições ambientais analisadas apresentaram valores fora da zona de conforto térmico para as linhagens Cobb e Ross, ou seja, o ambiente em que o experimento foi desenvolvido é considerado inapropriado.

As respostas fisiológicas das aves, durante o experimento, indicam a existência continuada de estresse térmico em todas as fases de criação.

\section{Conflito de Interesse}

Os autores declaram não existir conflito de interesse.

\section{Comitê de Ética}

O projeto de pesquisa foi aprovado pelo comitê de ética no uso de animais da Universidade Federal do Acre (UFAC), sob o número 91/2015.

\section{Referências}

Abreu, P.G.; Abreu, V.M.N.; Coldebella, A.; Hassemer, M.J.; Tomazelli, I.L. Medidas morfológicas em função do peso e da idade da ave, por meio de imagens. Revista Brasileira de Engenharia Agrícola e Ambienta, 16(7): 795-801, 2012.

Amaral, A.G.; Yanagi Junior, T.; Lima, R.R.; Teixeira, V.H.; Schiassi, L. Efeito do ambiente de produção sobre frangos de corte sexados criados em galpão comercial. Arquivo Brasileiro de Medicina Veterinária e Zootecnia, 63(3): 649-658, 2011.

Aviagen $囚$. Ross Manual de manejo de frangos de corte. 2018. Disponível em: <http://pt.aviagen.com/assets/Tech_Center/BB _Foreign_Language_Docs/Portuguese/RossBroilerHandbook2018-PT.pdf>. Acesso em: 17 nov. 2019.

Baêta, F.C.; Souza, C.F. Ambiência em edificações rurais - conforto animal. Viçosa: UFV, 2010. 269p.

Banzato, D.A.; Kronka, S.N. Experimentação agrícola. $4^{\text {a }}$ ed. Jaboticabal: Funep, 2006. 237p.

Borges, A.S.; Maiorka, A.; Silva, A.V.F. Fisiologia do estresse calórico e a utilização de eletrólitos em frangos de corte. Ciência Rural, 33(5): 975-981, 2003.

Bridi, A.M. Efeitos do ambiente tropical sobre a produção animal. 2010. Disponível em:
<http://www.uel.br/pessoal/ambridi/Bioclimat ologia_arquivos/EfeitosdoAmbienteTropicalso breaProducaoAnimal.pdf>. Acesso em: 17 jan. 2017.

Brossi, C.; Contreras-Castillo, C.J.; Amazonas, E.A.; Menten, J.F.M. Estresse térmico durante o pré-abate em frangos de corte. Ciência Rural, 39(4): 1296-1305, 2009.

Bueno, J.P.R.; Nascimento, M.R.B.M.; Carvalho, M.C.; Silva, M.C.A.; Silva, P.L.A.P.A. Características de termorregulação em frangos de corte, machos e fêmeas, criados em condições naturais de temperatura e umidade. Enciclopédia Biosfera, 10(19): 437-447, 2014.

Buffington, D.E.; Collazo-Arocho, A.; Canton, G.H.; Pitt, D.; Thatcher, W.W.; Collier, R.J. Black globe humity index (BGHI) as a comfort equation for dairy cows. Transactions of the ASAE, 24(3): 711-714, 1981.

Camargo, J.R.; Silva, I.O.; Nazareno, A.C.; Vieira, F.M.C.; Castro, A.C.; Dias, R.A.P. Qualidade de pintos em função do microclima, tempo de espera e idade de matrizes. Revista Brasileira de Engenharia Agrícola e Ambiental, 19(11): 1079-1085, 2015.

Cangar, Ö.; Aerts, J.-M.; Buyse, J.; Berckmans, D. Quantification of the Spatial Distribution of Surface Temperatures of Broilers. Poultry Science, 87: 2493-2499, 2008.

Cassuce, D.C. Determinação das faixas de conforto térmico para frangos de corte de diferentes idades criados no Brasil. 2011. 91 f. Tese (Doutorado em Engenharia Agrícola) Universidade Federal de Viçosa, 2011.

Cobb-Vantress. Cobb500. Suplemento de nutrição e desempenho do frango de corte. 2018. Disponível em: <https://www.cobbvantress.com/assets/Cobb-

Files/99b0cf062c/61bd2490-56d1-11e9-bfbd7963ec6b06e5.pdf>. Acesso em: 17 nov. 2019.

Costa, J.H.S.; Saraiva, E.P.; Santos, L.D. Efeito do ambiente sobre indicadores fisiológicos na produção de frangos de corte. Revista Verde de Agroecologia e Desenvolvimento Sustentável, Mossoró, 7(4): 54-58, 2012.

Cotta, T. Frangos de corte criação, abate e comercialização. $2^{\text {a }}$ ed. Viçosa: Aprenda Fácil, 2012. 243p.

Ferreira, D.F. Sisvar: a Guide for its Bootstrap procedures in multiple comparisons. Ciência e Agrotecnologia, 38(2): 109-112. 2014. 
Furlan, R.L. Influência da temperatura na produção de frangos de corte. In: Simpósio Brasil Sul de Avicultura, 7, 2006, Chapecó. Anais... Chapecó: SBA, 104-135, 2006.

Giloh, M.; Shinder, D.; Yahav, S. Skin surface temperature of broiler chickens is correlated to body core temperature and is indicative of their thermoregulatory status. Poultry Science, 91: 175-188, 2012.

Gomes, R.C.C.; Yanagi Junior, T.; Lima, R.R.; Yanagi, S.N.M.; Carvalho, V.F.; Damasceno, F.A. Predição do índice de temperatura do globo negro e umidade e do impacto das variações climáticas em galpões avícolas climatizados. Ciência Rural, 41(9): 16451651, 2011.

Guerra-Galdo, E.H.; Sanz, S.C.; Barber, F.E.; López-Jiménez, P.A. CFD model for ventilation assessment in poultry houses with different distribution of windows. International Journal of Energy and Environment, 6(5): 411-424, 2015.

Köppen, W. Grundriss der klimakunde. Berlin: W. Guyter, 1931. 390p.

Lin, H.; Zhang, H.F.; Du, R.; Gu, X.H.; Zhang, Z.Y.; Buyse, J.; Decuypere, E. Thermoregulation responses of broiler chickens to humidity at different ambient temperatures. II. Four weeks of age. Poultry Science, 84: 1173-1178, 2005.

Marchini, C.F.P.; Silva, P.L.; Nascimento, M.R.B.M.; Tavares, M. Frequência respiratória e temperatura cloacal em frangos de corte submetidos à temperatura ambiente cíclica elevada. Archives of Veterinary Science, 12(1): 41-46, 2007.

Moura, G.G.C.; Neto, L.F.; Santana, A.P.L. Melhoramento genético em aves de corte. Conexão Eletrônica,14(1): 363-369, 2017.

Nascimento, G.R.; Pereira, D.F.; Nääs, I.A.; Rodrigues, L.H.A. Índice fuzzy de conforto térmico para frangos de corte. Engenharia Agrícola Jaboticabal, 31(2): 219-229, 2011.

Nascimento, G.R.; Nääs, I.A.; Baracho, M.S.; Pereira, D.F.; Neves, D.P. Termografia infravermelho na estimativa de conforto térmico de frangos de corte. Revista Brasileira de Engenharia Agrícola e Ambiental, 18(6): 658-663, 2014.

Nascimento, M.R.B.M.; Bueno, J.P.R.; Olivieri, C.L.; Alves, R.L.O.R.; Rezende, F.M. Características da termorregulação antes e após diferentes tempos de exposição ao calor em frangos de corte. Enciclopédia Biosfera, Centro Científico Conhecer, 11(22): 525, 2015.

Nazareno, A.C.; Pandorfi, H.; Almeida, G.L.P.; Giongo, P.R.; Pedrosa, E.M.R.; Guiselini, C. Avaliação do conforto térmico e desempenho de frangos de corte sob regime de criação diferenciado. Revista Brasileira de Engenharia Agrícola e Ambiental, 13(6): 802-808, 2009.

Pires, M.F.A; Campos, A.T. Modificações ambientais para reduzir o estresse calórico em gado de leite. EMBRAPA, Juiz de Fora, MG, 2004.

Richards, S.A. The significance of changes in the temperature of the skin and body core of the chicken in the regulation of heat loss. Journal of Physiology, 216: 1-10, 1971.

Rostagno, H.S.; Albino, L.F.T.; Donzele, J.L.; Gomes, P.C.; Oliveira, R.F.; Lopes, D.C.; Ferreira, A.S.; Barreto, S.L.T. Tabelas brasileiras para aves e suínos: composição de alimentos e exigências nutricionais. Viçosa, MG: Universidade Federal de Viçosa, 2005. 186p.

Souza, E.D.; Souza, B.B.; Souza, W.H.; Cezar, M.F.; Santos, J.R.S.; Tavares, G.P. Determinação dos parâmetros fisiológicos e gradiente térmico de diferentes grupos genéticos de caprinos no semi-árido. Ciência e Agrotecnologia, 29(1): 177-184, 2005.

Tinôco, I.F.F.; Gates, R.S. Ambiência e construções para matrizes pesadas. In: Maccari, M.; Mendes, A.A. (Ed.) Manejo de matrizes de corte. Campinas: FACTA, 2005. p. 11-33. 